\title{
Focusing of multi-MeV, subnanosecond proton bunches from a laser-driven source
}

\author{
D. Jahn, ${ }^{1}$ D. Schumacher, ${ }^{2}$ C. Brabetz, ${ }^{2}$ F. Kroll, ${ }^{3,4}$ F. E. Brack, ${ }^{3,4}$ J. Ding, ${ }^{1}$ R. Leonhardt, ${ }^{1}$ \\ I. Semmler, ${ }^{1}$ A. Blažević, ${ }^{2,5}$ U. Schramm, ${ }^{3,6}$ and M. Roth ${ }^{1}$ \\ ${ }^{1}$ Institut für Kernphysik, Technische Universität Darmstadt, \\ Schlossgartenstraße 9, 64289 Darmstadt, Germany \\ ${ }^{2}$ GSI Helmholtzzentrum für Schwerionenforschung, Planckstraße 1, D-64291 Darmstadt, Germany \\ ${ }^{3}$ Helmholtz-Zentrum Dresden-Rossendorf, Bautzner Landstraße 400, 01328 Dresden, Germany \\ ${ }^{4}$ Technische Universität Dresden, Mommsenstrasse 13, 01062 Dresden, Germany \\ ${ }^{5}$ Helmholtz-Institut Jena, Helmholtzweg 4, 07734 Jena, Germany \\ ${ }^{6}$ Technische Universität Dresden, 01062 Dresden, Germany
}

(Received 16 October 2018; published 14 January 2019)

\begin{abstract}
We report on our latest transverse focusing results of subnanosecond proton bunches achieved with a laser-driven multi-MeV ion beamline. In the frame of the LIGHT collaboration, a target normal sheath acceleration (TNSA) source based $6 \mathrm{~m}$ long beamline was installed. In the past years, the laser-driven proton beam was transported and shaped by this beamline. The particle beam is collimated with a pulsed high-field solenoid and rotated in longitudinal phase space with a radio-frequency cavity which leads to an energy compression with an energy spread of $(2.7 \pm 1.7) \%\left(\Delta \mathrm{E} / \mathrm{E}_{0}\right.$ at $\left.\mathrm{FWHM}\right)$ or a time compression to the subnanosecond regime. Highest peak intensities in the subnanosecond regime open up an interesting field for several applications, e.g., proton imaging, as injectors in conventional accelerators or precise stopping power measurements in a plasma. We report on achieving highest peak intensities using an installed second solenoid as a final focusing system in our beamline to achieve small focal spot sizes. We measured a focal spot size of $1.1 \times 1.2 \mathrm{~mm}$ leading to $5.8 \times 10^{19}$ protons per s $\mathrm{cm}^{2}$ at a central energy bin of $(9.55 \pm 0.25) \mathrm{MeV}$, which can be combined with a bunch duration below 500 ps at FWHM.
\end{abstract}

DOI: 10.1103/PhysRevAccelBeams.22.011301

\section{INTRODUCTION}

Multi-MeV ion acceleration in a relativistic plasma driven by high-intensity ultrashort laser pulses became an intensively studied research field. Laser-driven ion beams offer small source sizes $(\sim 5 \mu \mathrm{m})$, short duration at the source ( $\sim$ ps), low emittance [1] and accelerating field gradients in the order of $\mathrm{MeV} / \mu \mathrm{m}$. Nevertheless, these sources deliver ion bunches with a broad energy spectrum and a large divergence angle, embedded in a large background radiation environment [electromagnetic pulse (EMP), x-rays, electrons]. These sources are intensively studied. At the same time, one of the main goals is to control the divergence and shape the beam.

In this context, the Laser Ion Generation, Handling and Transport (LIGHT) collaboration [3] was founded based on common interests and covers the expertise on the topics of laser and target shaping, simulations, ion transport as well

Published by the American Physical Society under the terms of the Creative Commons Attribution 4.0 International license. Further distribution of this work must maintain attribution to the author(s) and the published article's title, journal citation, and DOI. as postacceleration. The goal of the collaboration is to realize a TNSA-based test beamline for collimated, $\mathrm{MeV}$ ion bunches with only a few percent energy spread and make these shaped bunches usable for applications, such as: (i) the study of a laser-driven multi-MeV ion beamline (ii) providing an additional compact ion beamline for plasma physics at FAIR (iii) the use as a diagnostic tool for ultra-short, time-resolved proton imaging [4] (iv) the exploration of warm dense matter (WDM) via isochoric heating through the high particle numbers in the $\mathrm{MeV}$ region in short timescales (v) the investigation of energy loss measurements within a plasma with a precise time resolution in the subnanosecond regime.

In the upcoming years, the collaboration will concentrate its research on the energy deposition of ions in a dense plasma. This research topic aims at the key process in inertial confinement fusion (ICF) [2]. Unfortunately, stopping power measurements in a plasma are scarce and mostly suffer from time-averaging effects [5]. The plasma physics group at GSI performed several experiments regarding this topic in the past [5-7]: Two laser systems, PHELIX and nhelix, generate a plasma through a two-sided irradiation of a thin target leading to a full target ionization after 6-7 ns. This plasma is probed with a pulsed ion beam 
from the UNILAC accelerator (Universal Linear Accelerator at GSI). The energy loss of the beam ions is measured via the time-of-flight (TOF) method. While the plasma expands on the nanosecond regime, the UNILAC is operated at $108 \mathrm{MHz}$ delivering ion bunches with a pulse width between 3 and $4 \mathrm{~ns}$ and $10^{5}$ particles/per microbunch in a bunch period of $9.2 \mathrm{~ns}$. If the UNILAC ion beam is focused to $1 \mathrm{~mm}$ (limited by the beamline architecture) and passes an aperture of $500 \mu \mathrm{m}$ containing $10^{3}-10^{4}$ particles/per microbunch before reaching the laser-generated, uniform plasma. In this case, the UNILAC beam achieves a peak flux of $10^{3}-10^{4}$ protons $/\left(\mathrm{cm}^{2} \mathrm{~s}\right)$. In comparison, the laser ion source provides a peak flux which is higher by more than five orders of magnitude. The use of the UNILAC beam leads to a time-averaging effect as the density profile of the plasma changes during this interaction process. With the LIGHT beamline ion bunches below 500 ps with higher particle numbers are generated and they will reduce the timeaveraging effect. The working point at maximum stopping power at $<1 \mathrm{MeV} / \mathrm{u}$ is desirable for these measurements and is in the capability of the LIGHT beamline. On the way toward these measurements, a final focusing system to focus the beam transversely is necessary to provide the essential peak intensities including sufficient particle numbers and bunch durations on the subnanosecond regime. In recent UNILAC experiments, the measured time-of-flight signal had to be amplified due to the low particle numbers [5]. Higher particle numbers provide enough signal without a necessary amplification leading to an improved data acquisition.

We report on the progress in ion beam shaping towards these ion stopping measurements. In the past campaigns, we demonstrated the capture and the rotation in longitudinal phase space of the laser-driven proton beam (reported by S. Busold et al. [12]). In order to enable the necessary intensities for applications, the crucial goal was to focus transversely the beam to a small spot size. The final available beam parameters are necessary for the planning of advanced experiments. The topic of this work is the demonstration of a complete laser-driven beamline with focusing capabilities of a target point $6 \mathrm{~m}$ from source.

\section{SETUP AND DIAGNOSTICS OF THE LIGHT BEAMLINE}

The LIGHT beamline is located at the Z6 experimental area at GSI Helmholtzzentrum für Schwerionenforschung $\mathrm{GmbH}$. It consists of a laser-driven ion source based on the TNSA mechanism, a pulsed high-field solenoid, a radiofrequency (rf) cavity and an additional target chamber including a second high-field solenoid at the end. The second solenoid as a final focusing system replaced the previously used two quadrupole doublets [12]. Figure 1 shows the scheme of the LIGHT beamline setup located at GSI. The beamline is usually operated at a proton central energy of $8.5 \mathrm{MeV}$.

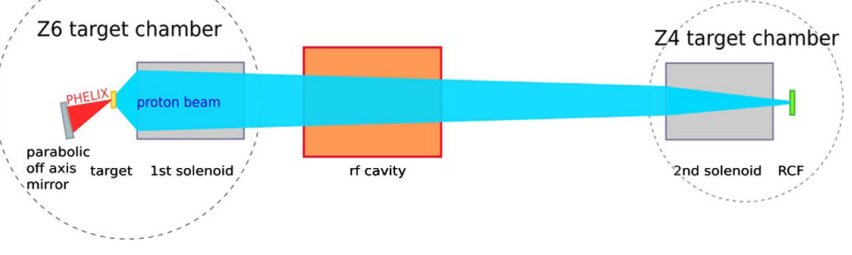

FIG. 1. Setup of the current LIGHT beamline at GSI Helmholtzzentrum für Schwerionenforschung: The PHELIX laser system (red) is compressed to several hundreds of femtoseconds and drives the TNSA source inside the Z6 target chamber. In the next step, the ion bunch is collimated with a high-field solenoid (grey) and transported into the rf cavity (red). The ions are further transported into a Z4 target chamber with a second high-field solenoid (grey) for transverse focusing. Moreover, diagnostics (radiochromic films, Thomson parabolas, diamond detectors) and devices for application studies (e.g., secondary targets) are placed inside this chamber or along the beamline.

The Petawatt High-Energy Laser for Heavy Ion Experiments (PHELIX) at GSI is a Nd:glass laser system with a $1053 \mathrm{~nm}$ wavelength providing pulse durations of $650 \mathrm{fs}$ [8]. The system delivers a $6 \times 10^{19} \mathrm{~W} / \mathrm{cm}^{2}$ intense laser beam with a focal spot size of $3.5 \mu \mathrm{m}$ diameter driving the TNSA source. For the experiment, a $10 \mu \mathrm{m}$ thin gold foil was used as a target and we measured an exponentially decaying proton spectrum up to $28 \mathrm{MeV}$ and the proton beam has a divergence up to 30 degrees (half angle).

The solenoid captures up to one third of the protons in the selected energy range via chromatic focusing with a large energy spread of about 20\% [9,10]. The propagation through the beamline over a few meters distance broadens the bunch to several nanoseconds because of this energy spread. The captured protons are transported to an rf cavity at a distance of $2.5 \mathrm{~m}$ to the target. The cavity enables a phase rotation in longitudinal phase space. The rf cavity is a three gap spiral resonator operating at $108.4 \mathrm{MHz}$ which can apply an electric potential of more than $\pm 1 \mathrm{MV}$ to the bunch. Particles entering the rf cavity at a different phase experience an acceleration or deceleration. At a certain $\mathrm{rf}$ input power the proton bunch is succesfully energy-compressed at a central energy of $8.5 \mathrm{MeV}$ with a narrow energy spread of $2.7 \%$ [11]. Increasing the rf input power leads to an "overrotation" of the proton bunch, so that the bunch length shortens to a minimum bunch duration in the subnanosecond regime [12]. In recent experiments, the transported proton bunch was compressed to a bunch length of $(458 \pm 40)$ ps at FWHM using a fast diamond detector [4]. These results were published by S. Busold et al. [12].

\section{FINAL TRANSVERSE FOCUSING SYSTEM}

At the end of the LIGHT beamline, a second pulsed high-field solenoid of the same design as the collimating one was installed inside the Z4 target chamber which 
enables to focus the beam transversely leading to the achievement of highest peak intensities.

The comparative beam transport simulations were performed with the TRACEWIN software developed by the CEA direction at IRFU in France [13]. The code calculates the beam dynamics in linear accelerator structures using ballistic particle tracking. The beam is modeled by its second order momentum or its macroparticle distribution, while the different accelerator elements are given by analytical expressions or field maps.

The on-site TNSA source spectrum was measured with radiochromic films (RCFs) and defined the macroparticle distribution for the simulation. The drift components, solenoids and cavity structures as well as field maps are part of the simulation. The TRACEWIN code has been used in previous studies, where it successfully reproduced experimental measurements by Busold et al. [12]. The simulations allow to determine the settings of all components (magnetic field strength, rf phase, rf amplitude) to transport ions successfully to the Z4 target chamber and focus transversely with the final focusing system. Dependent on the adjusted magnetic field of the second solenoid, the central energy of $8.5 \mathrm{MeV}$ is focused. The focal spot size is predicted to be $0.6 \times 0.6 \mathrm{~mm}$ at FWHM. Figure 2 shows the simulated focal spot.

The energy-dependent transverse proton beam profile was measured using radiochromic imaging spectroscopy [14]. This method is based on dosimetry films. It covers the whole lateral beam profile and enables a spectral and a spatial resolution. Several calibrated RCFs are aligned in a stacked configuration. The interaction with radiation changes the film colour corresponding to the deposited dose value. The protons are stopped at certain depth inside the RCF stack dependent on their energy and deposit the major part of their energy at this depth due to their Bragg peak behavior. The RCF films consist of sensitive layers and substrate layers. The RCF stack configuration for this study is as follows: $2 \times E B T 3,2 \times H D-V 2,2 \times E B T 3$ films produced by Gafchromic. Each $E B T 3$ layer consists

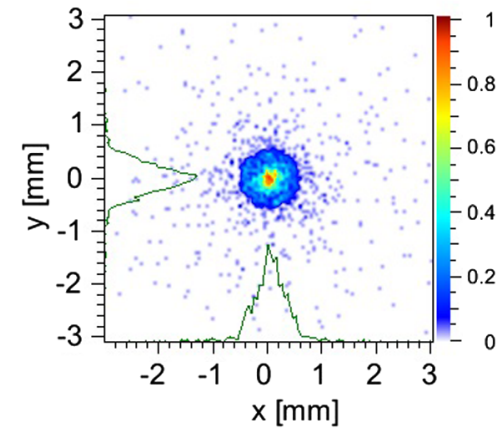

FIG. 2. The performed TRACEWIN simulation predicts a transverse focus at $5.5 \mathrm{~m}$ distance from the source with a focal spot size of $0.6 \mathrm{~mm} \times 0.6 \mathrm{~mm}$ at FWHM. The central energy $E_{0}$ is at the position 0 . of a $28 \mu \mathrm{m}$ thin active layer between two $120 \mu \mathrm{m}$ inactive polyester foils. The $H D-V 2$ layer contains an active $8 \mu \mathrm{m}$ active layer followed by a $97 \mu \mathrm{m}$ inactive polyester layer.

The characteristic Bragg peak behavior of the protons inside the RCF stack can be modeled with the Monte-Carlo simulation code SRIM [15]. Based on this model, the energy deposition and RCF response are calculated which is necessary for the analysis. Hereby, the initial proton energy bin with the highest energy loss within the sensitive layer of the specific RCF type is defined as the so called central energy. The corresponding energy deposition of protons within this stack and the central energies in each layer are shown in Fig. 3. The RCF detector was positioned $10 \mathrm{~cm}$ behind the second solenoid.

Regarding the solenoid's setting, the control of its magnetic field amplitude allows to choose the desired energy and the solenoid influences the particle trajectories: The distance of the focal spot is energy-dependent. In this beamtime, the first solenoid was set to $7.8 \mathrm{~T}$ leading to a collimation of the central energy over $6 \mathrm{~m}$ distance from target. For this measurement of the focal spot size, the rf cavity was not operated so a broad energy range was transported. The second high-field solenoid was operated at $5.6 \mathrm{~T}$ so that protons with a central energy of $(9.55 \pm$ $0.25) \mathrm{MeV}$ are focused on the RCF stack in $10 \mathrm{~cm}$ distance to the second solenoid. The received RCF raw data can be seen in Fig. 4. In this shot, the smallest, most intense focus lies in the 5th RCF layer. In this layer, the central energy is $(9.55 \pm 0.25) \mathrm{MeV}$.

Based on a calibration and the response function of the RCF films, the spatial beam profile with its energy spectrum is reconstructed [14]: Protons with lower energies are stopped in the previous layers, while protons with

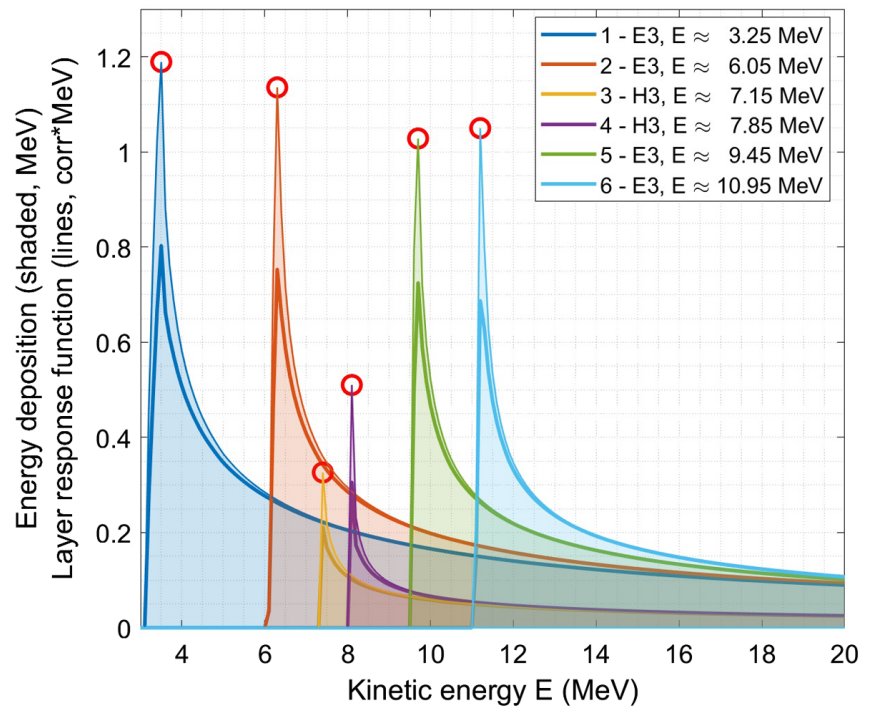

FIG. 3. Energy deposition and RCF response of protons in the active layers of the designed stack configuration. The so-called Bragg energies to the corresponding RCF layers are given in the legend. 


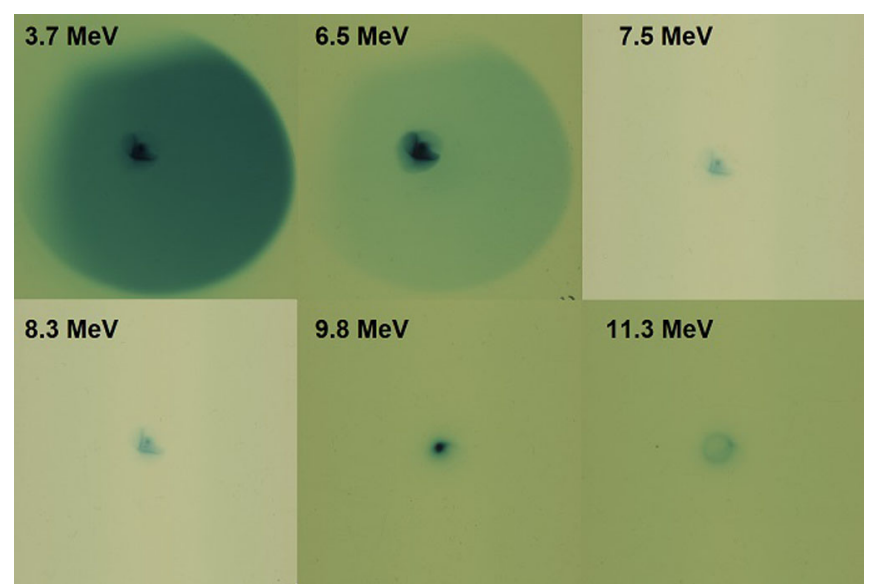

FIG. 4. For the detection of the focus, RCF films were placed $10 \mathrm{~cm}$ behind the second high-field solenoid. The central energy is written in black. The 3rd and 4th RCF layers are type $H D-V 2$, the others are type EBT3. In this shot, protons within a central energy bin of $(9.55 \pm 0.25) \mathrm{MeV}$ were focused on the 5 th RCF layer.

higher energies penetrate through the RCF layer, deposit a certain amount of energy in each layer and are stopped in the rear layers. Each RCF film is attributed to a $0.5 \mathrm{MeV}$ energy bin and main deposition of a certain energy is observed in the Bragg peak. First, the absolute particle number in the last layer based on the energy deposition response function (compare Fig. 3). Second, the proton number in the second last layer is calculated by subtracting the deposited energy of the protons, which were stopped in the last layer and which were stopped in this layer. This procedure is repeated for the other layers until the particle numbers for each layer are determined. In doing so, the integration is carried out over the whole spatial distribution in each film. As a result, the energy-dependent proton distribution is received.

For this case, the deconvolution of the measured RCF films leads to the reconstructed spectrum in Fig. 5. The beam is collimated by the first solenoid, transported through the beamline and focused with the second solenoid. The beam collimation around the central energy is demonstrated by the simulation code and by the RCF measurement. The total absolute proton number is $3.5 \times 10^{8}$. While the first solenoid collimates around the central energy of $8.5 \mathrm{MeV}$ (compare Fig. 5), the second solenoid focuses the proton bunch at the energy of $9.55 \mathrm{MeV}$. The focus of the second solenoid is observed in the 5th RCF layer (compare Fig. 4). Within this particle spectrum, the elliptical focal spot size has a value of $1.1 \times$ $1.2 \mathrm{~mm}$ at FWHM. The conversion of the deposited dose into absolute particle numbers per pixel is shown in Fig. 4. Within this layer at the energy of $(9.55 \pm 0.25) \mathrm{MeV}$, the focus of $1.1 \times 1.2 \mathrm{~mm}(\mathrm{FWHM})$ contains $2.5 \times 10^{7}$ protons in this energy range. The measured transverse beam profile

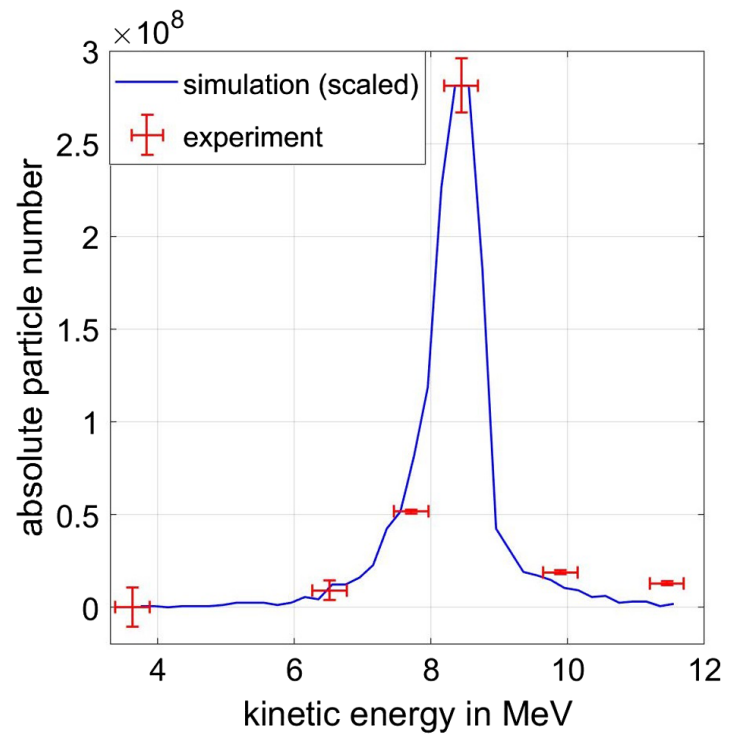

FIG. 5. Simulated and measured spectrum: For each RCF layer, the stopped absolute particle number within this layer is shown (red). Due to the operation of the first solenoid, the protons are collimated at the central energy. For comparison, the performed scaled TRACEWIN simulation results are displayed (blue).

is shown in Fig. 6. This corresponds to a proton intensity of $1.2^{7}$ protons $/\left(\mathrm{mm}^{2} \mathrm{MeV}\right)$.

This is necessary to fulfill the beam requirements for some applications. In future, both elements (the rf cavity and the second solenoid system) will be operated simultaneously. Using a proton bunch duration of $(458 \pm 40)$ ps, a beam current of $7.8 \times 10^{8} \quad 1 / \mathrm{ns} \simeq 124 \mathrm{~mA}$ will be generated. This equals $5.8 \times 10^{19}$ protons per s $\mathrm{cm}^{2}$ or a beam intensity of $7.42 \times 10^{7} \mathrm{~W} / \mathrm{cm}^{2}$.

The comparison of the measurement with the TRACEWIN simulations shows consistent behavior: Increasing the magnetic field of the solenoid, the chosen specific energy range is focused nearer to the solenoid. The difference in the focal spot size between the TraceWin simulation $(0.6 \times 0.6 \mathrm{~mm}$ at $\mathrm{FWHM})$ and the measurement $(1.1 \times 1.2 \mathrm{~mm}$ at FWHM $)$ has various reasons: The measurement was limited by the RCF resolution and it might be possible that the focused energy was stopped in an inactive layer.

Finally, we positioned a pinhole with a $1 \mathrm{~mm}$ diameter at the focal plane of the solenoid of the laser-driven ion beamline to define a well-characterized proton source with sufficient particle numbers for stopping power measurements. The pinhole is necessary to limit the beam according to the plasma expansion. An RCF stack in $15 \mathrm{~cm}$ distance to the pinhole detected the transmitted particles. The analysis of the RCF stack lead to $(1.7 \pm 0.9) \times 10^{7}$ protons at the central energy of $(9.7 \pm 0.25) \mathrm{MeV}$. This particle number is by a factor $10^{3}$ higher than the typically used conventional UNILAC beam. Through a better alignment of the pinhole exactly 


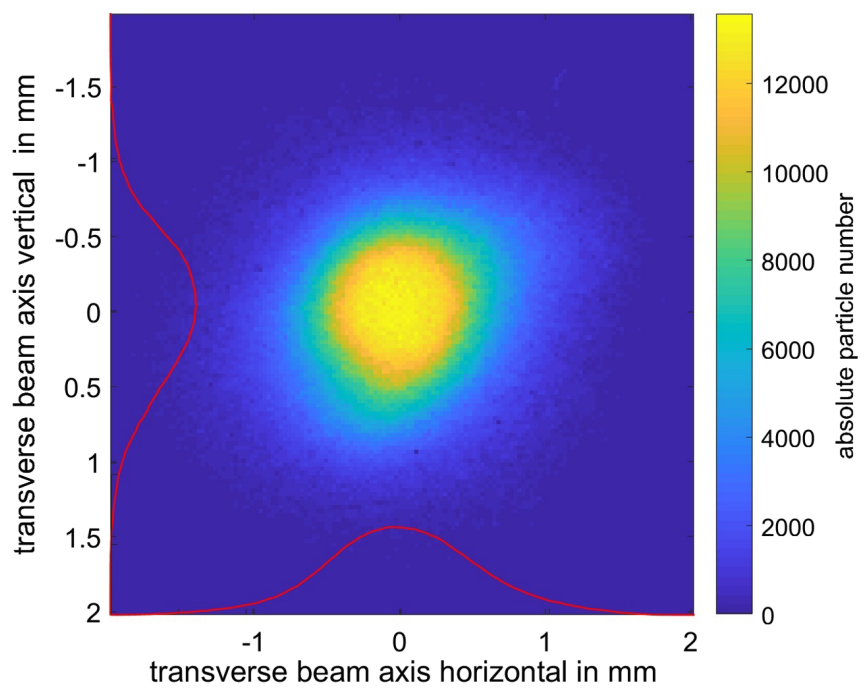

FIG. 6. Proton beam focus measured on the 5th RCF film (compare Fig. 4) with central energy of $(9.55 \pm 0.25) \mathrm{MeV}$ : The focal spot size is $1.1 \times 1.2 \mathrm{~mm}$ at FWHM. The deposited energy in this layer is converted into particle numbers per pixel.

on the focal spot position, higher particle numbers will be possible in future.

\section{CONCLUSION AND OUTLOOK}

The LIGHT beamline combines a TNSA source with conventional accelerator elements and delivers reproducibly multi-MeV, intense proton bunches in the subnanosecond regime. This article reports on the final focusing system and the final beam parameters which are crucial for applications in plasma physics and material sciences. The proton beam is transversely focused at the end of the beamline to a focal spot size of $1.1 \times 1.2 \mathrm{~mm}$ (FWHM) containing $(3.5 \pm 0.22) \times 10^{8}$ protons. In future, advanced experiments will be possible based on the final focusing system combined with the generation of a temporal focus $(458 \pm 40) \mathrm{ps}$ leading to a beam current of $7.8 \times 10^{8}$ protons $/ \mathrm{ns} \simeq 124 \mathrm{~mA}$.

Parallel to the presented work on proton beam shaping, we studied the generation and the transport of a mid- $\mathrm{Z}$ ion beam (e.g., carbon, fluorine) using ohmic heating. In the upcoming beamtime, the rotation of mid- $\mathrm{Z}$ ions in longitudinal phase space will be studied to achieve mid- $Z$ ion bunches in the nanosecond regime.

While protons possess a low stopping power, mid-Z ions are ideal for investigations of the energy loss within a plasma: In the next beamtime, the time-of-flight (ToF) measurement setup will be extended: The extended setup will be based on two diamond detectors that are sequentially traversed by the beam. If material is placed or a plasma is generated between the fast two diamonds, this will result in a later arrival time of the ion bunch at the second detector. For the plasma generation a second local high energy laser system can be used: while the PHELIX laser keeps the main driver for the LIGHT beamline, a second laser system, the nanosecond-high-energy laser for ion beam experiments (nhelix), can be used to irradiate a carbon foil enabling first energy loss studies in a plasma using a laser-driven, subnanosecond beam. The LIGHT ion beam offers in comparison to standard conventional accelerators higher particle intensities and ion bunches in the subnanosecond regime resulting in an enhancement of the measurement precision.

\section{ACKNOWLEDGMENTS}

The authors are thankful specially for the support of the PHELIX laser group and the HF group at GSI for supporting the realization. Special thanks belong to Michael Träger and Mladen Kis from the GSI Detector Laboratory and Gabriel Schaumann from the Technische Universität Darmstadt target laboratory. We appreciate the work of the high-field group at HZDR for the solenoid construction. LIGHT is partly funded by the ARD program of the Helmholtz Association.

[1] T. E. Cowan et al., Ultralow Emittance, Multi-MeV Proton Beams from a Laser Virtual-Cathode Plasma Accelerator, Phys. Rev. Lett. 92, 204801 (2004).

[2] M. Roth, T. E. Cowan, M. H. Key, S. P. Hatchett, C. Brown, W. Fountain, J. Johnson, D. M. Pennington, R. A. Snavely, S. C. Wilks, K. Yasuike, H. Ruhl, F. Pegoraro, S. V. Bulanov, E. M. Campbell, M. D. Perry, and H. Powell, Fast Ignition by Intense Laser-Accelerated Proton Beams, Phys. Rev. Lett. 86, 436 (2001).

[3] S. Busold et al., Shaping laser accelerated ions for future applications The LIGHT collaboration, Nucl. Instrum. Methods Phys. Res., Sect. A 740, 94 (2014).

[4] D. Jahn, M. Trger, M. Kis, C. Brabetz, D. Schumacher, A. Blazevic, M. Ciobanu, M. Pomorski, U. Bonnes, S. Busold, F. Kroll, F.-E. Brack, U. Schramm, and M. Roth, Chemical-vapor deposited ultra-fast diamond detectors for temporal measurements of ion bunches, Rev. Sci. Instrum. 89, 093304 (2018).

[5] W. Cayzac, A. Frank, A. Ortner, V. Bagnoud, M. Basko, S. Bedacht, C. Blser, A. Blazevic, S. Busold, O. Deppert, J. Ding, M. Ehret, P. Fiala, S. Frydrych, D. O. Gericke, L. Hallo, J. Helfrich, D. Jahn, E. Kjartansson, and M. Roth, Experimental discrimination of ion stopping models near the Bragg peak in highly ionized matter, Nat. Commun. 8, 15693 (2017).

[6] A. Ortner, A. Frank, A. Blazevic, and M. Roth, Role of charge transfer in heavy-ion-beam-plasma interactions at intermediate energies, Phys. Rev. E 91, 023104 (2015).

[7] D. Schumacher, S. Bedacht, A. Blazevic, S. Busold, W. Cayzac, A. Frank, T. Hessling, D. Kraus, A. Ortner, G. Schaumann, and M. Roth, Temperature measurement of hohlraum radiation for energy loss experiments in indirectly laser heated carbon plasma, Phys. Rev. E 96, 043210 (2017). 
[8] V. Bagnoud et al., Commissioning and early experiments of the PHELIX facility, Appl. Phys. B 100, 137 (2010).

[9] V. Kumar, Understanding the focusing of charged particle beams in a solenoid magnetic field, Am. J. Phys. 77, 737 (2009).

[10] S. Busold, D. Schumacher, O. Deppert, C. Brabetz, S. Frydrych, F. Kroll, M. Joost, H. Al-Omari, and A. Blažević, B. Zielbauer, I. Hofmann, V. Bagnoud, T. E. Cowan, and M. Roth, Focusing and transport of high-intensity multi-MeV proton bunches from a compact laser-driven source, Phys. Rev. ST Accel. Beams 16, 101302 (2013).

[11] S. Busold, D. Schumacher, O. Deppert, C. Brabetz, F. Kroll, and A. Blažević, V. Bagnoud, and M. Roth, Commissioning of a compact laser-based proton beam line for high intensity bunches around $10 \mathrm{MeV}$, Phys. Rev. ST Accel. Beams 17, 031302 (2014).
[12] S. Busold, D. Schumacher, C. Brabetz, D. Jahn, F. Kroll, O. Deppert, U. Schramm, T. E. Cowan, and A. Blažević, v. Bagnoud, and M. Roth, Towards highest peak intensities for ultra-short MeV-range ion bunches, Sci. Rep. 5, 12459 (2015).

[13] D. Uriot and N. Pichoff, TraceWin documentation (CEA Saclay, Saclay, 2011).

[14] F. Nürnberg, M. Schollmeier, E. Brambrink, A. Blazevic, D. C. Carroll, K. Flippo, D. C. Gautier, M. Geissel, K. Harres, B. M. Hegelich, O. Lundh, K. Markey, P. McKenna, D. Neely, J. Schreiber, and M. Roth, Radiochromic film imaging spectroscopy of laser-accelerated proton beams, Rev. Sci. Instrum. 80, 033301 (2009).

[15] J. F. Ziegler, SRIM, http://www.srim.org (2013). 